\title{
Outcomes in Adolescents Undergoing Treatment for Drug-Resistant Tuberculosis in Cape Town, South Africa, 2008-2013
}

\author{
Sizulu Moyo ${ }^{1, *}$; Jennifer J Furin ${ }^{2}$; Jennifer Hughes ${ }^{1}$; Johnny Daniels ${ }^{1}$; Leigh Snyman ${ }^{1}$; \\ Odelia Muller ${ }^{1}$; Vivian Cox $^{1}$; Amir Shroufi ${ }^{1}$; Helen Cox $^{3}$ \\ ${ }_{1}^{1}$ Medecins Sans Frontieres Khayelitsha, Cape Town, South Africa \\ ${ }^{2}$ Division of Infectious Diseases and HIV Medicine, Case Western Reserve University, Cleveland, USA \\ ${ }^{3}$ Faculty of Health Sciences, University of Cape Town, Cape Town, South Africa \\ ${ }^{*}$ Corresponding author: Sizulu Moyo, Medecins Sans Frontieres Khayelitsha, Cape Town, South Africa. Tel: +27-213645490, Fax: +27-213617051, E-mail: msfocb-khayelitsha-drtb-epi@ \\ brussels.msf.org; sizulumoyo@gmail.com
}

Received: January 30, 2014; Revised: March 1, 2014; Accepted: March 15, 2014

\begin{abstract}
Background: There is limited data on outcomes of adolescents with drug-resistant tuberculosis (DR-TB).
Objectives: To describe patient outcomes and factors associated with outcomes of adolescents diagnosed with DR-TB in Khayelitsha, South Africa.

Patients and Methods: A retrospective analysis of data for adolescents aged 10-19 years who were diagnosed with DR-TB between January 2008 and August 2013 was conducted. The proportions of adolescents with treatment success (cure and treatment completion), failure of treatment, those lost from treatment, and those who died were calculated and compared by HIV status. Proportions and odd ratios are presented.

Results: Seventy-one adolescents were diagnosed with DR-TB. Six (8\%) were lost to care before treatment could be initiated. The median age of those started on treatment was 18 years(IQR 15.8-18.9). Eighteen (27.7\%) were HIV infected. Of the 44 adolescents with final treatment outcomes, $36.4 \%(n=16)$ were successfully treated, $9.1 \%(n=4)$ died, $11.4 \%(n=9)$ failed treatment and $43.2 \%(n=19)$ were lost from treatment (treatment interrupted for $\geq 2$ consecutive months). Three of the four patients who died, died within two months of starting therapy. Loss from treatment, and treatment success (cure or treatment completion) did not differ between HIV infected and un-infected adolescents, OR:2.0,(95\% CI 0.56-7.50), $\mathrm{P}=0.27$; and $\mathrm{OR}: 1.2$ (95\% CI 0.37-4.43), $\mathrm{P}=0.71$, respectively. All five patients who failed treatment and one of those lost from treatment subsequently died. Overall mortality was 12.1/100 person years.

Conclusions: HIV infected and uninfected adolescents with DR-TB experienced poor outcomes with high proportions of mortality, treatment failure and loss from treatment. Mortality occurred early in the treatment period suggesting delayed presentation and/or diagnosis. Innovative and targeted strategies are needed to encourage early presentation and improve adherence to treatment among adolescents.
\end{abstract}

Keywords: Extensively Drug-Resistant Tuberculosis; Adolescent; Patient Outcome Assessment

\section{Background}

Drug-resistant tuberculosis (DR-TB) defined as strains of tuberculosis with any resistance to rifampicin, is a growing public health concern, across the world $(1,2)$. According to World Health Organization (WHO) estimates, there are more than 650,000 prevalent cases of DR-TB in the world (3). It is estimated that at least $10 \%$ of these occur among children (4), and although excellent treatment outcomes have been reported in pediatric populations (5), thousands of children die from this disease each year, largely due to lack of access to diagnosis and treatment $(6,7)$.

There has been a recent increased interest in pediatric DR-TB, with efforts being made to increase access to time- ly diagnosis and prompt initiation of care (8-10). While the pediatric population is often written about as a homogenous group, children along the age spectrum have different needs in terms of diagnosis and treatment that merit special attention (11). The period of adolescence defined by the WHO as between the ages of 10-19 years (12) may pose significant challenges to the management of DR-TB $(13,14)$. One of the only studies of DR-TB in adolescents was of HIV co-infected adolescents in Mumbai which found that poor treatment outcomes were common, with $64 \%$ experiencing death, treatment failure, or default (15).

There may be several factors related to the poor treat-

Implication for health policy/practice/research/medical education:

There is limited data on outcomes of adolescents with drug-resistant tuberculosis (DR-TB). This study shows poor outcomes in HIV infected and uninfected adolescents with DR-TB and highlights the need for adolescent specific interventions and support to encourage early presentation and retention on treatment.

Copyright (C) 2014, Pediartric Infections Research Center; Published by Kowsar Corp. This is an open-access article distributed under the terms of the Creative Commons Attribution License, which permits unrestricted use, distribution, and reproduction in any medium, provided the original work is properly cited. 
ment outcomes seen in adolescents with DR-TB. Studies of other diseases in this population have shown that adolescents face specific developmental challenges that may make adherence to complicated medical regimens difficult $(16,17)$. Second, adolescents may be experiencing rapid periods of growth that render dosing inadequate (18). Third, adolescents may not always engage well with standard services, which are usually adult orientated, and finally, adolescence is a time of major life changes such as geographic dislocation, finding work, developing significant relationships (e.g. long term commitments), that may also make treatment of chronic diseases more complicated (19-21).

\section{Objectives}

This paper reports on the treatment outcomes for a group of adolescents undergoing treatment for DR-TB in Khayelitsha, Cape Town, South Africa between 2008 and 2013. The outcomes for the entire cohort have been reported elsewhere in the literature (22). The adolescent outcomes are presented separately here given the poor treatment outcomes seen in the Mumbai cohort (15) and since this is to our knowledge one of the largest cohorts of adolescent patients reported in the literature.

\section{Patients and Methods}

Khayelitsha township is situated about $30 \mathrm{~km}$ outside Cape Town and has an estimated population of approximately 400000 (23) people. It has a high burden of HIV and TB with a $69 \%$ HIV/DR-TB confection rate in 2011 (22). Antenatal HIV prevalence was 33\% in 2010 (24). The TB case notification rate was $1500 / 100000$ in 2008 (25). A survey conducted in the same year found DR TB in $3.3 \%$ and $7.7 \%$ of new and previously treated TB cases respectively, and estimated DR-TB notification rate at 51/100,000/year $(25,26)$.

This study is a retrospective review of a cohort of adolescents who were diagnosed with DR-TB in Khayelitsha, South Africa between January 2008 and August 2013. The details of the entire DR-TB cohort in Khayelitsha and a description of the methodology have been described in detail elsewhere $(22,27)$. In summary, the following elements were added to the existing TB programme at primary care level: - DR-TB specific staff training and clinical support, DR-TB counseling, social assistance, programme supervision and evaluation, TB infection control support and advice, a local sub-acute short stay facility and specialist outreach services for pediatrics and audiometry screening (22). In addition to primary care services that include HIV testing and counselling, DR-TB services provided at the primary care clinics include nurse and medical officer consultation, sputum collection for diagnosis and treatment monitoring, DR-TB specific counselling, directly observed therapy (DOT) and administration of injectable drugs where they have been prescribed. The sub-acute facility is available for patients who do not need hospital care but require support to take their med- ication, deal with side effects, or overcome other difficulties to receiving treatment at home.

Drug susceptibility testing (DST) was initially only available for TB cases considered at high DR-TB risk, who were defined as patients who had been previously treated for $\mathrm{TB}$, those not responding to first-line TB treatment, close contacts of patients diagnosed with DR-TB, health care workers and those with a history of being in prison. From late 2011, all individuals with suspected TB underwent testing with the Xpert MTB/Rif assay $(28,29)$, a molecular based test for rapid and simultaneous detection of tuberculosis and rifampicin resistance endorsed by the WHO, and is being rolled out in South Africa $(28,29)$. A standard treatment regimen in line with the South African National Department of Health recommendations aligned to WHO recommendations was used $(29,30)$. This was adapted based on second line DST results or when treatment was failing. Available drugs included second line injectable agents (kanamycin, capreomycin), ethambutol, ethionamide and pyrazinamide, terizidone, fluoroquinolones (ofloxacin, moxifloxacin) and P- aminosalicylic acid (PAS), clofazimine, and high dose isoniazid. Treatment duration was for at least 18 months 29, 30 . Treatment outcome definitions follow WHO recommendations, as follows $(29,31)$ :

Cured: Treatment completed as recommended by the national policy without evidence of failure AND three or more consecutive cultures taken at least 30 days apart are negative after the intensive phase.

Treatment completed: Treatment completed as recommended by the national policy without evidence of failure BUT no record that three or more consecutive cultures taken at least 30 days apart are negative after the intensive phase.

Treatment success: The sum of cured and treatment completed.

Treatment failed: Treatment terminated or need for permanent regimen change of at least two anti-TB drugs because of: lack of culture conversion by the end of the intensive phase, bacteriological reversion in the continuation phase after conversion to negative, evidence of additional acquired resistance to fluoroquinolones or second-line injectable drugs, or adverse drug reactions (ADRs).

Died: A patient who dies for any reason during the course of treatment.

Lost to follow up: A patient whose treatment was interrupted for 2 consecutive months or more (previously defined as default (30).

\subsection{Data Collection and Analysis}

Routine DR-TB programme data was initially collected from paper registers at primary care clinics and entered into a MS Access database. From February 2013, data was directly imported from an electronic database of all DR-TB patients (EDR. Web), in the Khayelitsha district. 
Analyses were conducted using STATA v. 12.0 (Stata Corp, College Station, TX, USA). Continuous variables were compared using the Mann-Whitney test. Proportions were compared using with the Chi2 test. 95\% CI and p vales are presented. Evaluation of the Khayelitsha DR-TB program was approved by the University of Cape Town, Faculty of Health Sciences Human Ethics review committee (ref 540/2010).

Outcomes up to August 2013 were analysed. The proportion of adolescents with treatment success (cure and treatment completion), failure of treatment, those lost from treatment, and those who died were calculated and compared by HIV status. For this analysis, patients who interrupted treatment for $\geq 2$ consecutive months were defined as lost from treatment. Patients who did not return to care or were never traced after diagnosis were regarded as lost to follow up. Information on deaths was obtained from regular cross linkage of available civil identifiers with the national death registry, and from DR-TB counsellors who are active in the area and become aware of deaths among DR-TB patients.

Extensive drug-resistance (XDR) was defined as resistance to any fluoroquinolone and one of two second-line injectable drugs (capreomycin and kanamycin), in addition to multidrug resistance (31). Pre-extensive drug-resistance (pre-XDR TB) was defined as tuberculosis resistance to either any fluoroquinolone or one of two second-line injectable drugs (capreomycin andkanamycin), in addition to multidrug resistance (32).

\section{Results}

Seventy-one (71) adolescents, aged 10-19 years, median age 18 years (IQR 15.7-18.9), were diagnosed with DR-TB between January 2008 and August 2013 in Khayelitsha. Six of these (8\%) did not start treatment: two could not be traced after diagnosis, one out-migrated, two refused treatment, one is known to have died before treatment was started. There was no significant difference in age between the adolescents who started treatment and those who did not, $\mathrm{P}=0.8$. The demographic features of the 65 adolescents who started treatment are shown in Table 1. Treatment was started at a primary care clinic in $67.7 \%$ of these adolescents.

HIV co-infection was $27.7 \%$. There was no significant difference in HIV infection between adolescent males and females, OR: 0.4 (95\% CI 0.12-1.28), P = 0.13. Seventeen of the 18 (94\%) HIV infected adolescents were on antiretroviral therapy (ART) at the time of DR-TB diagnosis. Median CD4 count at diagnosis was 168 cells/ $/ \mathrm{mm}^{3}$ (IQR 97-457). Of the 12 adolescents with known resistance to second line drugs, 8 (66.7\%), had resistance to both an injectable agent (kanamycin) and a flouroquinolone (ofloxacin). Table 2 shows details of the six patients who did not start treatment. In all six patients this was their first episode of DR-TB, and none had resistance to second line agents. The patient known to have died before commencing treatment was HIV infected and died within a month of DR-TB diagnosis.

Table 1. Demographic Features of Adolescents Diagnosed and Started on DR-TB Treatment for 2008-2013 $(n=65)^{\text {a, b }}$

\begin{tabular}{|c|c|}
\hline Variable & Value \\
\hline Age, y, IQR & $18(15.8-18.9)$ \\
\hline \multicolumn{2}{|l|}{ Gender } \\
\hline Male & $29(45)$ \\
\hline Female & 36 \\
\hline \multicolumn{2}{|l|}{ HIV status } \\
\hline$+\mathrm{ve}$ & $18(27.7)$ \\
\hline -ve & $45(69.2)$ \\
\hline unknown & $2(3.1)$ \\
\hline \multicolumn{2}{|l|}{ Site of treatment initiation } \\
\hline Primary Care Clinic & $44(67.7)$ \\
\hline Subacute facility & $7(10.8)$ \\
\hline Hospital ${ }^{\mathrm{C}}$ & $14(21.5)$ \\
\hline \multicolumn{2}{|l|}{ Drug Susceptibility Testing pattern } \\
\hline Presumed MDR-TB & $1(1.5)$ \\
\hline Rifampicin mono resistance & $20(30.8)$ \\
\hline MDR \& no 2nd line resistance ${ }^{d}$ & $30(46.2)$ \\
\hline MDR \& 2nd line resistance ${ }^{d}$ & $12(18.5)$ \\
\hline MDR 2nd line resistance unknown ${ }^{d}$ & $2(3.1)$ \\
\hline \multicolumn{2}{|c|}{$\begin{array}{l}\text { a Abbreviations: IQR, Interquartile range; MDR-TB, multidrug resistant } \\
\text { tuberculosis. } \\
\mathrm{b}_{\text {Data are presented as No. (\%). }} \\
\mathrm{c}_{\text {District and specialist TB hospitals with specialist care services. }} \\
\mathrm{d}_{\text {MDR-TB -multidrug resistant tuberculosis (resistant to rifampicin }} \\
\text { and isoniazid). }\end{array}$} \\
\hline
\end{tabular}

\begin{tabular}{lccccc}
\hline Table 2. Patients & Who Did Not Commence Treatment $(\mathrm{n}=6)^{\mathrm{a}}$ & & \\
\hline Age, $\mathbf{G}$ & Gender & HIV Status & Resistance Profile & Previous TB History & Outcome \\
\hline $\mathbf{1 3 . 8}$ & female & positive & MDR TB-no second line resistance & none & LTFU (did not return to care and never found) \\
$\mathbf{1 5 . 3}$ & male & unknown & Rifampicin mono resistance & none & Out-migrated \\
$\mathbf{1 6 . 4}$ & female & negative & Rifampicin mono resistance & none & Refused treatment and LTFU \\
$\mathbf{1 8 . 1}$ & female & positive & MDR-TB-no second line resistance & none & LTFU (did not return to care and never found) \\
$\mathbf{1 8 . 5}$ & female & Positive & MDR-TB- no second line resistance & none & Died before start of treatment \\
$\mathbf{1 9 . 9}$ & male & unknown & MDR-TB-no second line resistance & none & Refused treatment and LTFU \\
\hline
\end{tabular}

a Abbreviations: LTFU, lost to follow up. 


\subsection{Treatment Outcomes}

Final treatment outcomes were available for 44 adolescents. (Three patients were transferred out of the area during treatment and 18 are still on treatment), Table 3. Treatment success was achieved in $36.4 \%$, $43 \%$ were lost from treatment, $9 \%$ died while on treatment and treatment failed in $11.4 \%$ (Table 3 ).

Among those who died, three died within two months of starting treatment, and one within four months. All four patients had resistance to second line drugs (three had XDR TB 31and one had pre-XDR TB 32), and were HIV negative. All the five patients in whom treatment failed are known to have subsequently died. All five were female, (age range 16-19 years), and all except one were HIV negative. Nineteen patients were lost from treatment, and among these one restarted treatment again, and one is known to have subsequently died. The median duration of treatment of those who were lost from treatment was 8.9 months (IQR 4.8-13.3).

Overall mortality $(\mathrm{n}=10)$ among those who started treatment was 12.1/100 person years, over the period of observation. Loss from treatment and treatment success (cure or treatment completion) was not significantly different between HIV infected and un-infected adolescents, OR: 2.0, (95\% CI 0.56-7.50), P = 0.27, and OR: 1.2 (95\% CI $0.37-4.43), \mathrm{P}=0.71$ respectively. Only one of the $12 \mathrm{pa}$ tients with known resistance to second line drugs was successfully treated.

\section{Discussion}

We observed poor treatment outcomes in the majority of adolescents undergoing treatment for DR-TB treatment in Cape Town with a treatment success rate of only $36 \%$. Nine percent of adolescents died while on treatment, while $11 \%$ experienced treatment failure, and $43 \%$ were lost from treatment. Treatment was not initiated in $8 \%$ of those diagnosed.

Our treatment success rate is comparable to that reported among HIV positive adolescents in India (36\% had favourable outcomes) (15). However we observed much lower mortality in a larger cohort of patients with final treatment outcomes 15 . Our loss from treatment proportion of $43 \%$ was 1.6 times that seen in the Mumbai cohort (15). It is possible that the high proportion of those

\begin{tabular}{lc}
\hline \multicolumn{2}{l}{ Table 3. Final Treatment Outcomes for Adolescents $(\mathrm{n}=44)^{\mathrm{a}}$} \\
\hline Final Outcomes & No. $(\%)$ \\
\hline Cured & $11(25)$ \\
Completed treatment & $5(11.4)$ \\
Treatment success & $16(36.4)$ \\
Failed treatment & $5(11.4)$ \\
Lost from treatment & $19(43.2)$ \\
Died & $4(9.1)$ \\
\hline
\end{tabular}

\footnotetext{
${ }^{\mathrm{a}}$ Data are presented as No. (\%).
}

lost from treatment masked some deaths in our cohort (only one of these is known to have died); therefore overall mortality is likely to be higher than presented here. Nonetheless, overall mortality over the period of observation was high, with $15.4 \%$ of all patients who started treatment and had treatment outcomes known to have died during the period of observation.

Six patients were lost to care before therapy could be initiated: - one died, while two refused treatment and one out-migrated from the area, and three were lost to follow-up. Analysis of the outcomes of the entire Khayelitsha DR-TB cohort found that the majority of patients who did not initiate treatment died prior to receipt of their diagnosis and thus initiation of treatment (22). In the present analysis only one adolescent is known to have died before treatment could be initiated. At inception of the decentralized Khayelitsha DR-TB programme in 2007, the median time from diagnosis to initiation of treatment was 54 days, time during which patients could experience further deterioration and/or death. In 2011 this delay had been reduced by $50 \%$ (27 days) and is expected to have been further reduced since the introduction of Xpert MTB/Rif testing for MDR-TB diagnosis in the district $(22,28)$. In Gauteng, South Africa, Xpert testing at pointof-care resulted in same day treatment initiation in all those with a positive result (33).

Two adolescents refused treatment, an observation previously reported in TB patients $(15,34)$. In this population this can be attributed to a myriad of reasons including the physical and emotional changes, and the pressures associated with life during this period. Adolescents have to contend with peer pressure, competing demands for time (school attendance, work and social activities), have to navigate relationships with parents, peers and partners, may have genuine difficulties in understanding complex treatment, or may believe that they do not need treatment, among many other reasons that have been cited as barriers to adherence to treatment for chronic diseases in this population $(13,21,35)$. These factors potentially contributed to the exceptionally high loss from treatment that we observed. Further research should therefore investigate strategies targeted at adolescent specific needs and challenges within DR-TB programs. A potential useful intervention to encourage acceptance of and adherence to treatment could be modeled along the "Youth group patient support" model aimed at decreasing loss from HIV and ART care among the youth that has been piloted in Khayelitsha, South Africa (36). In the pilot only $4 \%$ of patients had been lost to care over a period of 8 months (36).

Three of the four patients who died while on treatment died within two months of initiating treatment. These patients who had resistance to second line drugs and were HIV negative demonstrate very poor outcomes and early mortality in extensive resistance. They could however also be disease that presented and was diagnosed and 
treated late in its course as has been described in other settings $(15,37)$. In the Indian adolescent cohort, three of the four reported deaths occurred prior to treatment initiation and one within three weeks of initiation, and were ascribed to delayed diagnosis and treatment rather than treatment failure (15). The three adolescents who died while on treatment in study were diagnosed with DR-TB before the roll out of Xpert MTB/Rif testing, strongly supporting this hypothesis of delayed diagnosis rather than treatment failure.

The TB/HIV co-infection rate of $27.7 \%$ while lower than the $69 \%$ that has reported for the entire DR-TB cohort in Khayelitsha (22) shows a high HIV burden in this age group in our setting. This could have implications for treatment adherence since it has been shown a high pill burden and adverse drug effects are associated with loss from DR-TB treatment (38): treatment for both conditions entails a large number of tablets which have significant adverse drugs events which may overlap resulting in additive drug toxicities (39). However we found no difference in loss from treatment rates between HIV infected and un-infected adolescents in this analysis.

Our study had several limitations. First, it is based on routine DR-TB program data and is subject to limitations associated with such data, mainly that we may have missed some cases. However, these would be very few given the extensive monitoring and evaluation system and clinical support implemented as part of the decentralized program. Second, the cohort described is from a township in Cape Town and may not be generalizable to other populations. Lastly, were not able to analyse data on viral load in HIV infected patients. In spite of these limitations, the analysis has several important findings. It shows very poor outcomes among HIV un-infected and infected adolescents, with very high proportions of loss from treatment, treatment failure and mortality. It highlights the need for targeted interventions to support treatment adherence among adolescents a high risk group facing different challenges to those faced by adults and young children. Lastly it underscores the need for integration of HIV and DR-TB care among adolescents since a significant proportion of adolescents with DR-TB will be HIV infected in high HIV burden settings.

\section{Acknowledgements}

We are grateful to the healthcare staff in Khayelitsha, the City of Cape Town and Province of the Western Cape health departments for the tireless efforts in this program, and we acknowledge the adolescents affected by DR-TB in Khayelitsha.

\section{Authors' Contributions}

Study concept and design: Sizulu Moyo, Jennifer J Furin, $\mathrm{JH}$; Acquisition of data: Jennifer Hughes, Odelia Muller; Statistical analysis and interpretation of data: Sizulu Moyo; drafting of manuscript- Sizulu Moyo, Jennifer J
Furin, HC, Vivian Cox, Leigh Snyman, Helen Cox, Amir Shroufi; critical revision of manuscript: Sizulu Moyo, Helen Cox, Vivian Cox, AM; study supervision: Helen Cox, Vivian Cox, Leigh Snyman, Sizulu Moyo.

\section{Financial Disclosure}

The study was part of Medecins Sans Frontieres decentralised DR-TB programme in Khayelitsha, South Africa. No other funding was obtained.

\section{Funding/Support}

Implementation of the Khayelitsha DR-TB programme was funded by Medecins Sans Frontieres (MSF Belgium).

\section{References}

1. World Health Organization. Anti-tuberculosis drug resistance in the world. Report no. Geneva, Switzerland; 2008. Contract No.: 4.

2. Metanat M, Sharifi-Mood B, Shahreki S, Dawoudi SH. Prevalence of multidrug-resistant and extensively drug-resistant tuberculosis in patients with pulmonary tuberculosis in zahedan, southeastern iran. Iran Red Crescent Med J. 2012;14(1):53-5.

3. World Health Organization. Towards universal access to diagnosis and treatment of multidrug-resistant and extensively drug-resistant tuberculosis by 2015. Geneva, Switzerland; 2011.

4. World Health Organization. Global Tuberculosis Report 2012. Geneva, Switzerland; 2012.

5. Ettehad D, Schaaf HS, Seddon JA, Cooke GS, Ford N. Treatment outcomes for children with multidrug-resistant tuberculosis: a systematic review and meta-analysis. Lancet Infect Dis. 2012;12(6):449-56.

6. Perez-Velez CM. Pediatric tuberculosis: new guidelines and recommendations. Curr Opin Pediatr. 2012;24(3):319-28.

7. Tabarsi P, Mardani M. Extensively Drug-Resistant Tuberculosis: A Review Article. Arch Clin Infect Dis. 2012;7(3):81-4.

8. Seddon JA, Hesseling AC, Marais BJ, Jordaan A, Victor T, Schaaf HS. The evolving epidemic of drug-resistant tuberculosis among children in Cape Town, South Africa. Int J Tuberc Lung Dis. 2012;16(7):928-33.

9. Reubenson G. Pediatric drug-resistant tuberculosis: a global perspective: a global perspective. Paediatr Drugs. 2011;13(6):349-55.

10. World Health Organization. Roadmap for childhood tuberculosis: towards zero deaths. Geneva, Switzerland; 2013.

11. Seddon JA, Furin JJ, Gale M, Del Castillo Barrientos H, Hurtado RM, Amanullah F, et al. Caring for children with drug-resistant tuberculosis: practice-based recommendations. Am J Respir Crit Care Med. 2012;186(10):953-64.

12. World Health Organization. Adolescent Health. Available from: http://www.who.int/topics/adolescent_health/en/.

13. Geldenhuys H, Sorsdahl K, Kafaar F, Hatherill M, Hanekom WA, Stein DJ, et al. Risky behaviour and psychosocial correlates in adolescents - is there a link with tuberculosis? Afr J Psychiatry (Johannesbg). 2011;14(5):383-7.

14. Thampi N, Stephens D, Rea E, Kitai I. Unexplained deterioration during antituberculous therapy in children and adolescents: clinical presentation and risk factors. Pediatr Infect Dis J. 2012;31(2):129-33.

15. Isaakidis P, Paryani R, Khan S, Mansoor H, Manglani M, Valiyakath A, et al. Poor outcomes in a cohort of HIV-infected adolescents undergoing treatment for multidrug-resistant tuberculosis in Mumbai, India. PLoS One. 2013;8(7).

16. Greenley RN, Kunz JH, Biank V, Martinez A, Miranda A, Noe J, et al. Identifying youth nonadherence in clinical settings: data-based recommendations for children and adolescents with inflammatory bowel disease. Inflamm Bowel Dis. 2012;18(7):1254-9.

17. Nichols SL, Montepiedra G, Farley JJ, Sirois PA, Malee K, Kammerer B, et al. Cognitive, academic, and behavioral correlates of medication adherence in children and adolescents with perinatally 
acquired HIV infection. J Dev Behav Pediatr. 2012;33(4):298-308.

18. Chiang CY, Bai KJ, Lee CN, Enarson DA, Suo J, Luh KT. Inconsistent dosing of anti-tuberculosis drugs in Taipei, Taiwan. Int $J$ Tuberc Lung Dis. 2010;14(7):878-83.

19. Phongsamart W, Kitai I, Gardam M, Wang J, Khan K. A population-based study of tuberculosis in children and adolescents in Ontario. Pediatr Infect Dis J. 2009;28(5):416-9.

20. Fair CD, Sullivan K, Dizney R, Stackpole A. "It's like losing a part of my family": transition expectations of adolescents living with perinatally acquired HIV and their guardians. AIDS Patient Care STDS. 2012;26(7):423-9.

21. Hodgson I, Ross J, Haamujompa C, Gitau-Mburu D. Living as an adolescent with HIV in Zambia -- lived experiences, sexual health and reproductive needs. AIDS Care. 2012;24(10):1204-10.

22. Cox HS, Hughes J, Daniels J, Azevedo V, McDermid C, Poolman $\mathrm{M}$, et al. Community-based treatment of drug-resistant tuberculosis in Khayelitsha, South Africa. Int J Tuberc Lung Dis. 2014;18(4):441-8.

23. 2013. Available from: http://www.capetown.gov.za/en/ stats $2011 \% 20$ Census $\% 20 \% 20$ Health\%2 0District $\% 2$ OProfiles/ Khayelitsha\%20Health\%20District.pdf.

24. Western Cape Department of Health. Western Cape antenatal HIV survey. 2010.

25. Medecins Sans Frontieres. Khayelitsha 2001-2011, Activity Report: 10 years of HIV/TB care at primary health care level.; 2011.

26. Cox HS, McDermid C, Azevedo V, Muller O, Coetzee D, Simpson J, et al. Epidemic levels of drug resistant tuberculosis (MDR and XDR-TB) in a high HIV prevalence setting in Khayelitsha, South Africa. PLoS One. 2010;5(11).

27. Medecins Sans Frontieres. Scaling up diagnosis and treatment of drug-resistant tuberculosis in Khayelitsha, South Africa. 2011.

28. Stop TB Department, World Health Organization. Update: Implementation and roll-out of Xpert MTB/RIF. 2013.

29. National Department of Health. Management of Drug resistant
Tuberculosis. Policy guidelines. South Africa, Pretoria; 2011

30. World Health Organization. Guidelines for the programmatic management of drug-resistant tuberculosis: Emergency update 2008.Geneva: World Health Organization; 2008.

31. World Health Organization. Definitions and reporting framework for tuberculosis - 2013 revision; 2013.

32. Kim DH, Kim HJ, Park SK, Kong SJ, Kim YS, Kim TH, et al. Treatment outcomes and survival based on drug resistance patterns in multidrug-resistant tuberculosis. Am J Respir Crit Care Med. 2010;182(1):113-9.

33. Hanrahan CF, Selibas K, Deery CB, Dansey H, Clouse K, Bassett $J$, et al. Time to treatment and patient outcomes among TB suspects screened by a single point-of-care xpert MTB/RIF at a primary care clinic in Johannesburg, South Africa. PLoS One. 2013;8(6).

34. Wolman M, Reichman L. Tuberculosis patients: some don't. some do, some won't. some will. Lancet Infect Dis. 2007;7(3):17980.

35. Hanghoj S, Boisen KA. Self-reported barriers to medication adherence among chronically ill adolescents: a systematic review. J Adolesc Health. 2014;54(2):121-38.

36. Wilkinson L, Henwood R, Mkhosana N, Abrams S, Shuba Z, Patten G. Youth Groups: Linkage to and retention in ART care strategy in a youth specific primary care clinic, Khayelitsha. 6th South African AIDS conference; Durban. 2013.

37. Mitnick C, Bayona J, Palacios E, Shin S, Furin J, Alcantara F, et al Community-based therapy for multidrug-resistant tuberculosis in Lima, Peru. N Engl J Med. 2003;348(2):119-28.

38. Gler MT, Podewils LJ, Munez N, Galipot M, Quelapio MI, Tupasi TE. Impact of patient and program factors on default during treatment of multidrug-resistant tuberculosis. Int J Tuberc Lung Dis. 2012;16(7):955-60.

39. World Health Organization. Guidelines for the programmatic management of drug-resistant tuberculosis-2011 update. 2011. 